\title{
An Image Quality Comparison Study Between Homemade and Commercial Dental Cone-beam CT Systems
}

\section{Trang Thi Ngoc Tran}

National Yang-Ming University School of Dentistry

\section{David Shih-Chun Jin}

National Yang-Ming University School of Biomedical Science and Engineering

Kun-Long Shih

National Yang-Ming University School of Biomedical Science and Engineering

Ming-Lun Hsu

National Yang-Ming University School of Dentistry

Jyh-Cheng Chen ( $\nabla$ jyhcheng2010@gmail.com )

National Yang-Ming University School of Biomedical Science and Engineering

\section{Research Article}

Keywords: Cone-beam computed tomography (CBCT), system performance, image quality, spatial resolution, signal to noise ratio (SNR), contrast to noise ratio (CNR)

Posted Date: March 17th, 2021

DOI: https://doi.org/10.21203/rs.3.rs-165836/v1

License: (1) This work is licensed under a Creative Commons Attribution 4.0 International License.

Read Full License

Version of Record: A version of this preprint was published at Journal of Medical and Biological Engineering on November 15th, 2021. See the published version at https://doi.org/10.1007/s40846-02100663-7. 
Original article:

\title{
An image quality comparison study between homemade and commercial dental cone-beam CT systems (Running title: An image quality comparison study between two CBCT systems)
}

\author{
Trang Tran Thi Ngoc ${ }^{1}$, David Shih-Chun Jin², Kun-Long Shih ${ }^{3}$, Ming-Lun Hsu*1, Jyh-Cheng Chen*2,4,5 \\ ${ }^{1}$ Department of Dentistry, National Yang-Ming University, Taipei, Taiwan. \\ ${ }^{2}$ Department of Biomedical Imaging and Radiological Sciences, National Yang-Ming University, Taipei, \\ Taiwan. \\ ${ }^{3}$ Department of Electro-Optical Engineering, National Taipei University of Technology. Taipei, Taiwan. \\ ${ }^{4}$ Department of Medical Imaging and Radiological Technology, Yuanpei University of Medical Technology, \\ Hsinchu, Taiwan. \\ ${ }^{5}$ School of Medical Imaging, Xuzhou Medical University, Jiangsu, China \\ *Correspoding author: \\ Jyh-Cheng Chen. Email: jyhcheng2010@gmail.com \\ Ming-Lun Hsu. Email: mlhsu@ym.edu.tw \\ (Contribute equally to this paper.)
}

First author: Trang Tran Thi Ngoc

Contact: Ms. Trang Tran Thi Ngoc, email: ngoctrangrhm09@gmail.com.

${ }^{1}$ Department of Dentistry, National Yang-Ming University, Taipei, Taiwan.

${ }^{2}$ Department of Biomedical Imaging and Radiological Sciences, National Yang-Ming University, Taipei, Taiwan. 


\begin{abstract}
Purpose: Cone-beam computed tomography (CBCT) has been widely applied in dental and maxillofacial imaging. Several dental CBCT systems have been recently developed in order to improve the performance. This study aimed to evaluate the image quality of our prototype (YMU-DENT-P001) and compare with another commercial CBCT system made in Taipei, Taiwan (system A).

Methods: The Micro-CT-Contrast Scale, Micro-CT Water and Micro-CT HA phantoms were utilized to evaluate the contrast to noise ratio (CNR), signal to noise ratio (SNR), uniformity, distortion, and the linear relation between image intensity and calcium hydroxyapatite concentrations. Another customized thin-wire phantom was used to evaluate the FWHM spatial resolution. These phantoms were scanned by the two CBCT systems using the same exposure protocol. Acquired data were evaluated using the image analysis platform designed by our lab, which followed ISO standard.

Results: The SNR of our prototype YMU-DENT-P001 is nearly five times higher (prototype: $159.85 \pm 3.88$; A: $35.42 \pm 0.61$; $\mathrm{p}<0.05$ ) while CNR is three times higher than system A (prototype: $329.39 \pm 5.55$; A: $100.29 \pm 2.31$; $p<0.05$ ). The spatial resolutions recorded are $0.2446 \mathrm{~mm}$ in the prototype DENT and $0.5179 \mathrm{~mm}$ in the commercial system A. Image produced by the prototype is also better in terms of distortion level $(0.03 \mathrm{~mm}$ in prototype, compared to $0.294 \mathrm{~mm}$ in $\mathrm{A}, \mathrm{p}<0.05)$. The linear relationship between bone mineral density (BMD) and image intensity of both systems were similarly performed.
\end{abstract}

Conclusion: Within the limitation of this study, our prototype DENT-P001 has potential to produce better image quality than system A in terms of spatial resolution, SNR, CNR, uniformity, and distortion.

Keywords: Cone-beam computed tomography (CBCT), system performance, image quality, spatial resolution, signal to noise ratio (SNR), contrast to noise ratio (CNR). 


\section{Declarations}

+ Funding: This research instrument building was supported by funding from the Ministry of Science and Technology, Taiwan, R.O.C grants: MOST 107 - $2328-8-010-003$.

+ Conflicts of interest: The authors have no conflict of interest to disclose.

+ Data Availability Data available on request from the authors.

+ Code Availability Not applicable.

+Author Contribution Statement: All authors read and approved the final manuscript. All authors contributed to the study conception and design:

\begin{tabular}{|l|l|}
\hline \multicolumn{1}{|c|}{ Author name } & \multicolumn{1}{c|}{ Contribution } \\
\hline Trang Thi Ngoc Tran & $\begin{array}{l}\text { Design the experiment, design customized phantom, collect data, } \\
\text { analysis, writing manuscript. }\end{array}$ \\
\hline David Shih-Chun Jin & $\begin{array}{l}\text { Design the experiment, collect data, software for image analysis, data } \\
\text { interpretation. }\end{array}$ \\
\hline Kun-Long Shih & $\begin{array}{l}\text { Collect data from experiment, analyze system A's parameters and } \\
\text { images quality. }\end{array}$ \\
\hline Jyh-Cheng Chen & $\begin{array}{l}\text { Supervisor, define the concept of the work, design the experiment, } \\
\text { review manuscript. }\end{array}$ \\
\hline Ming-Lun Hsu & Supervisor, design the experiment, review manuscript. \\
\hline
\end{tabular}

+ Classification code: JEL I10 


\section{Introduction}

1.1. Cone-beam computed tomography:

Cone-beam computed tomography (CBCT) was invented in the second half of 1990s and has been widely applied in dental and maxillofacial imaging, which helps physicians to have multi-planar views of targeted anatomical or abnormal structure $[1,2]$. Some investigations concluded that $\mathrm{CBCT}$ provide comparable image quality and visibility of structures while better bone segmentation than multislice computed tomography (MSCT) with adequate energy set up [3-5]. In clinical practice, the quality of an image can be assessed in relation with the visibility, such as differentiation between various tissues, detection of root fracture of the teeth or bone fracture, signs of pathology, cortical bone thickness or trabecular bone's structure assessment. Those visualizations can be quantitatively assessed using some parameters regarding image characteristics of dental images: spatial resolution, contrast sensitivity, and noise [6,7]. They are inter-dependent and should always be considered together in assessment of image quality.

\subsection{Quantitative assessment of the image quality}

Image quality is defined by many factors regarding imaging hardware, energy set up, image acquisition and reconstruction steps [6]. Spatial resolution is the ability to distinguish between small and discrete objects or structures, which appreciates the fine details in an image. Some authors mentioned spatial resolution as sharpness that can be affected by the two-dimensional detector (size, number and spacing of detector elements), the three-dimensional reconstruction process, the size of the X-ray focal spot, the source-object-detector distances, reconstruction filter and reconstructed voxel size [6-8]. As the anatomical structure in dental maxillofacial region is complex and some small details (ex: periodontal ligament gap, root fracture, bone fracture, etc.) is required to present for analysis and treatment planning, thus spatial resolution is an important factor that clinicians care about.

Contrast in radiographic imaging is the ability to differentiate various types of materials having different attenuation coefficients. Image contrast is related to many factors as physical contrast of objects or materials, exposure factor, the bit depth of reconstructed image and display setting (such as window/level) in the image visualization stage [7]. Compared to conventional medical CT systems, which have a very high contrast sensitivity, CBCT have lower reliability on mineral density measurement or differentiation between different kinds of soft tissues [9]. The grey 
scale in CBCT is also represented in reported intensity values which different from CT contrast scale in conventional CT system [10]. Moreover, the image pixel values among different manufacturers are reported in different scales, which makes it difficult to quantitatively compare the quality image in term of contrast sensitivity.

Image noise implies to the random variability in voxel values, which appears as graininess on CT images and compromises the visibility of the object, particularly relating to low-contrast tissues. By increasing acquisition set up which relates to the number of X-ray photons such as scanning time, tube amperage, and peak kilovoltage, the noise can be impaired. Besides, in the reconstruction stage, some smoothing filters can also be applied for reducing noise. In practical context, a lesion-to-background contrast is related to the contrast-to-noise ratio (CNR) which has been considered to be more closely related to image quality than image noise [11]. The higher the CNR and SNR, the better the image quality.

This study aims to compare image quality of two CBCT systems: commercial system A (Taipei, Taiwan) and our prototype YMU-DENT-P001 dental CBCT system (developed by Department of Biomedical Imaging and Radiological Sciences - National Yang-Ming University, Taiwan) using quantitative assessment approach.

\section{Materials and methods:}

2.1 Phantoms and image acquisition:

Four different phantoms were utilized in this study in order to quantitatively assess the image quality: QRM Micro-CT water phantom, QRM Micro - contrast scale phantom, Micro-CT HA phantom (QRM Quality Assurance in Radiology and Medicine $\mathrm{GmbH}$, Möhrendorf, Germany) and a customized thin-wire phantom (Table 1).

All of these phantoms were scanned by the two systems under same exposure conditions: $60 \mathrm{kVp}$ of tube voltage and $2 \mathrm{mAs}$ of tube current. The phantoms were placed at the isocentre. Some differences in term of technical information between system A and Prototype DENT-P001 are listed in Table 2. 
Table 1: Different phantoms used for image quality assessment [12]:

\begin{tabular}{|l|l|l|l|}
\hline Phantom & Contained materials & \multicolumn{1}{c|}{$\begin{array}{l}\text { Purpose of } \\
\text { use }\end{array}$} \\
\hline $\begin{array}{l}\text { QRM Micro- } \\
\text { CT water } \\
\text { phantom }\end{array}$ & $\begin{array}{l}\text { DNR } \\
\text { Uniformity } \\
\text { Distortion }\end{array}$ \\
\hline $\begin{array}{l}\text { QRM Micro- } \\
\text { CT Contrast }\end{array}$ & & & \\
\hline $\begin{array}{l}\text { Scale phantom } \\
\text { CT Micro- } \\
\text { phantom HA }\end{array}$
\end{tabular}

Note: HA: Hydroxyapatite; PMMA: Poly methyl methacrylate; SNR: signal to noise ratio; CNR: contrast to noise ratio; BMD: Bone mineral density.

\subsection{Method of analysis:}

Acquired data were used to evaluate image quality (IQ) using the analysis platform designed by our lab, which followed standard ISO157082:2002/EN16016-3 about operation and interpretation of CT non-destructive testing [13]. The CT image analysis protocol included five steps: 
+ Setting data loading format: input data, define volume of interest (VOI);

+ Window level control: automatically adjusted so that the images of the two systems are displayed comparably;

+ Image viewer for multislice: central or nearly central slice is chosen;

+ Analysis function setup: include list of analytical options

+ Results exported: show the results

Several parameters were determined to interpret quantitative IQ including: SNR, CNR, uniformity, distortion (roundness), diameter, linearity, and spatial resolution.

2.2.1. Signal to noise ratio (SNR), uniformity (U) and distortion (roundness and diameter):

Water phantom was used to calculate SNR, uniformity and distortion. The central or nearly central slice of the phantom was utilized for region of interest (ROI) analysis. Five featureless circular ROIs were chosen (in the center and near the center) in which the diameter of each ROI accounts for $20 \%$ of the diameter of the phantom (Figure 1).

SNR was determined as the ratio of the average and standard deviation of all pixel intensity values in each ROI.

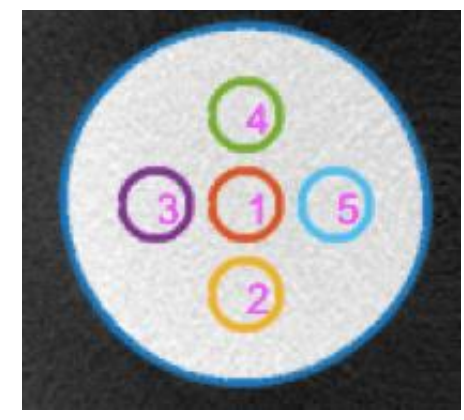

$$
\begin{gathered}
\mathrm{SNR}_{R O I(i)}=\frac{\mu_{R O I(i)}}{\sigma_{R O I(i)}}, \quad i \text { from } 1 \text { to } 5 \\
\mathrm{SNR}=\frac{\sum_{1}^{5} \operatorname{SNR}_{R O I(i)}}{5}
\end{gathered}
$$

Fig. 1: Regions of interest for signal to noise ratio (SNR) \& uniformity's anaslysis: from 1 to 5; for distortion determination: outline blue circle 
Table 2: Comparison of technical parameters and reconstruction algorithms between two dental CBCT systems:

\begin{tabular}{|c|c|c|}
\hline & System A & Prototype DENT-P001 \\
\hline SOD & $350 \mathrm{~mm}$ & $430 \mathrm{~mm}$ \\
\hline OID & $170 \mathrm{~mm}$ & $190 \mathrm{~mm}$ \\
\hline Rotation angle: & $\begin{array}{l}\text { Start angle: } 135 \\
\text { Rotation angle: } 268\end{array}$ & $\begin{array}{l}360 \text { full rotation with angle } \\
\text { sampling: } \\
\text { + } 2 \text { degree: QRM phantom } \\
\text { +1 degree: customized phantom }\end{array}$ \\
\hline Sensor size & $\begin{array}{l}\text { Horizontal: } 960 \text { pixel } \\
\text { Vertical: } 786 \text { pixel }\end{array}$ & $\begin{array}{l}\text { Horizontal: } 2176 \text { pixel } \\
\text { Vertical: } 1192 \text { pixel }\end{array}$ \\
\hline $\begin{array}{l}\text { Sensor element } \\
\text { size }\end{array}$ & $0.15 \mathrm{~mm}$ & $0.139 \mathrm{~mm}$ \\
\hline Binned input & 1 & 1 \\
\hline Voxel size & $0.125 \mathrm{~mm}$ & $0.096 \mathrm{~mm}$ \\
\hline FOV & $\begin{array}{l}\text { Diameter: } 98 \mathrm{~mm} \\
\text { Height: } 80 \mathrm{~mm}\end{array}$ & $\begin{array}{l}21.97 \mathrm{~cm} \text { (horizontal) * } 138 \mathrm{~cm} \\
\text { (vertical) }\end{array}$ \\
\hline X-ray source & $60-90 \mathrm{kV}, 2-10 \mathrm{~mA}$ & $50-90 \mathrm{kV}, 2-10 \mathrm{~mA}$ \\
\hline Focal spot & $0.5 \mathrm{~mm}$ (Toshiba, Japan) & $0.5 \mathrm{~mm}$ (PDM, Spellman, US) \\
\hline Detector & CMOS & CMOS \\
\hline Bit depth & $>14$ bit & 16 bit \\
\hline Source filtration & $2.6 \mathrm{~mm}$ Alumina & $3 \mathrm{~mm}$ Alumina \\
\hline $\begin{array}{l}\text { Reconstruction } \\
\text { algorithm }\end{array}$ & Analytical: FDK algorithm. & Analytical: FBP algorithm. \\
\hline $\begin{array}{l}\text { Pre- } \\
\text { reconstruction } \\
\text { calibration }\end{array}$ & $\begin{array}{l}\text { flood field correction; dark-field } \\
\text { correction; gain correction; X-ray } \\
\text { exposure uniformity; center of } \\
\text { rotation alignment }\end{array}$ & $\begin{array}{l}\text { flood field, dark field correction, } \\
\text { rigid body alignment. }\end{array}$ \\
\hline $\begin{array}{l}\text { Post- } \\
\text { reconstruction } \\
\text { correction }\end{array}$ & $\begin{array}{l}\text { Energy-related attenuation coefficient } \\
\text { based HU correction; ring-artifact } \\
\text { reduction, outlier-voxel removal } \\
\text { (noise, spark points are included) and } \\
\text { circle mask-out of FOV region to be } \\
\text { zero, and metal artifact reduction. }\end{array}$ & $\begin{array}{l}\text { dynamic HU calibration, noise } \\
\text { reduction, ring reduction and metal } \\
\text { artifact reduction algorithm. }\end{array}$ \\
\hline Software kernel & $\begin{array}{l}\text { self-developed software integrated by } \\
\text { open source package to proof of } \\
\text { concept on python platform (TomoPy } \\
\text { with ASTRA package). }\end{array}$ & $\begin{array}{l}\text { AVRG }- \text { a commercialized } \\
\text { algorithm supplied by Orimtech } \\
\text { (Illinois, USA). }\end{array}$ \\
\hline
\end{tabular}

Note: SOD: Source to Object distance; OID: Object to Detector distance; FOV: Field of view; CMOS: Complementary mental oxide semiconductor; FBP: Filtered backprojection; FDK: Feldkamp-Davis-Kress; HU: Hounsfield unit. 
The uniformity of each ROI was expressed by:

$$
\mathrm{U}=\frac{\mu_{\max }-\mu_{\min }}{\mu_{\max }+\mu_{\min }} * 100 \%
$$

, where $\mu_{\max }$ and $\mu_{\min }$ is a maximum and minimum pixel intensity value, respectively. Average uniformity value of five ROIs was then calculated.

Diameter was determined as circumference of the outline ROI.

The deformity level of the reconstructed image was defined as roundness following conventions ISO-1011 (Figure 2):

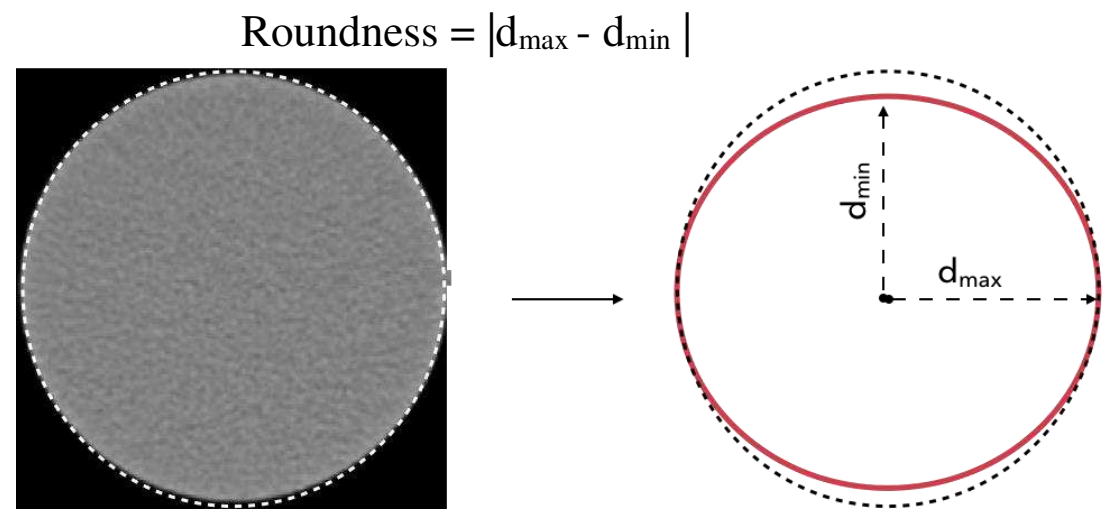

Fig. 2: Roundness calculation

2.2.2. Linearity relationship between image intensity and density

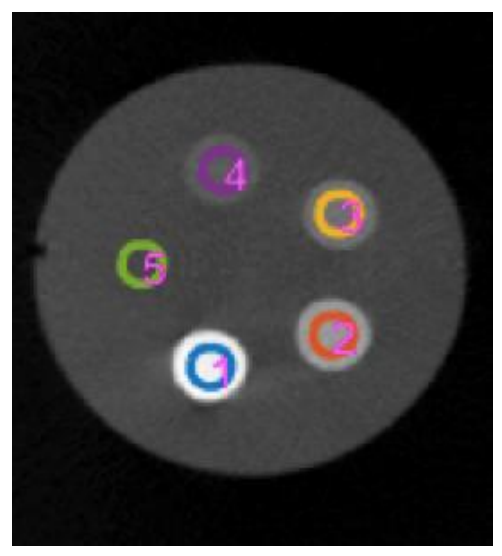

Fig. 3: Regions of interest of each hydroxyapatite concentration inserts

QRM Micro-CT HA phantom contains five cylindrical inserts with different HA concentration ( 0 , $50, \quad 200, \quad 800$ and $1200 \mathrm{mg}$ $\mathrm{HA} / \mathrm{cm} 3)$. The chosen ROI of each HA concentration is a homogenous area within the test cylindrical and accounts for $90 \%$ of each reconstructed circle's area. The ROI diameter was 49 pixels (4.704 $\mathrm{mm})$ and 38 pixels $(4.75 \mathrm{~mm})$ in prototype DENT-P001 and system A, respectively (Figure 3). The range and mean of pixel value of each ROI were recorded. 


\subsubsection{Contrast to noise ratio $(\mathrm{CNR})$}

Central slice of reconstructed QRM Contrast scale phantom was chosen. The ROI diameter was 52 pixels $(4.992 \mathrm{~mm}$ ) in prototype DENT-P001 and 40 pixels (5 $\mathrm{mm}$ ) in system A. Average intensity value was calculated in the center as well as the background. The expression of CNR follows 16016-3:2011 standard (Figure 4):

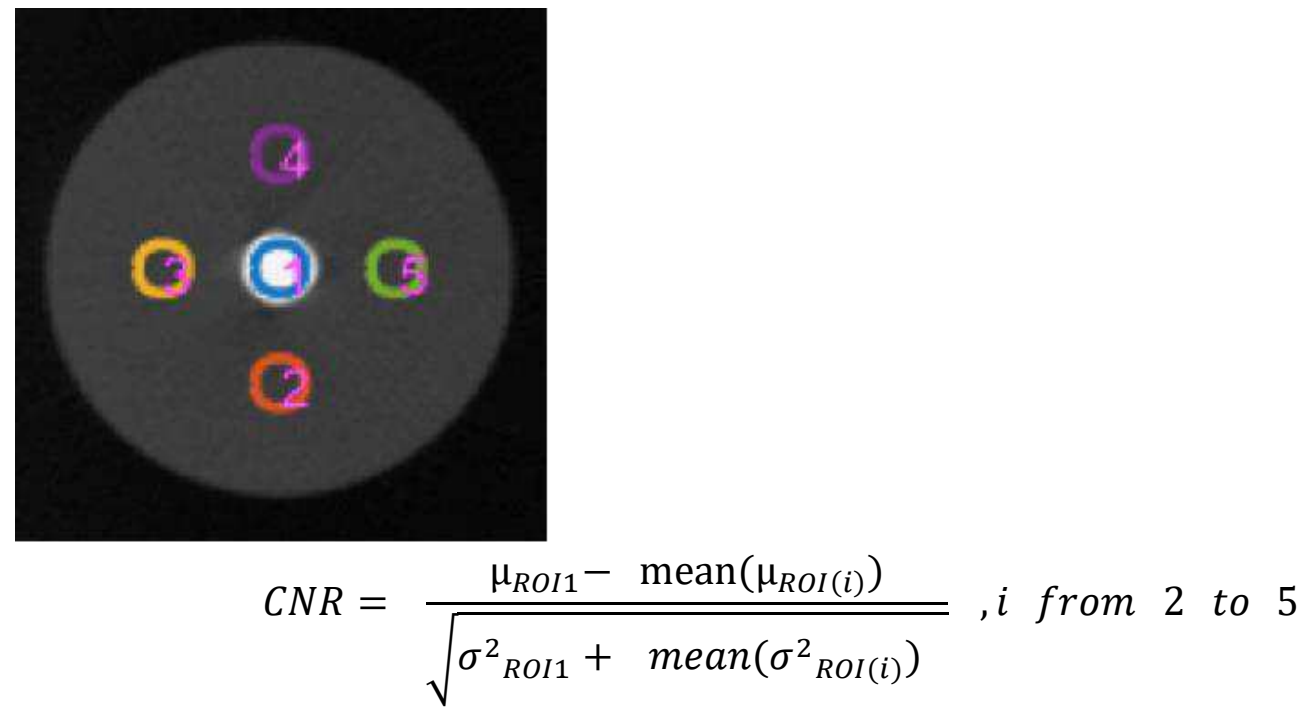

Fig. 4: Contrast to noise determination

\subsubsection{Spatial resolution}

Customized wire phantom is built as a rectangular parallelepiped. It contains many different sizes of wire which are inserted in epoxy resin and aligned parallel to the phantom axis of rotation. Point spread function (PSF) can be investigated using analysis platform designed by our lab. PSF is obtained by the distribution of the CT value of pixels along the reconstructed image's midline vertically and horizontally. Gaussian fitting was then applied and the full width at half maximum (FWHM) was calculated as representation for spatial resolution.

\subsection{Statistical analysis}

The analysis was implemented in horizontal slices, but did not evaluate along the z-axis. We repeated this procedure in 10 central continuous reconstructed slices for each measurement. t-test was used to examine the differences between the two CBCT systems. Results were considered statistically significant for $p<0.05$. 
Statistical analyses were performed using R Statistical software version 3.3.3.

\section{Results}

3.1. SNR, uniformity, diameter, and roundness:

Results of analysis are summarized in Table 3. SNR and uniformity's value of our prototype dental CBCT system are significantly higher than commercial system A $(p<0.05)$. These results can prove that the image quality in terms of noise in prototype DENT-P001 image is better than that of commercial system A.

Diameter of the phantom is $32 \mathrm{~mm}$, while reconstructed image of the phantom edge is $33.5903 \pm 0.004 \mathrm{~mm}$ in system A and $33.1437 \pm 0.005 \mathrm{~mm}$ in Prototype. Moreover, the level of distortion or roundness in system $\mathrm{A}$ is higher than our prototype's result $(0.294 \mathrm{~mm}$ compared to $0.03 \mathrm{~mm})(\mathrm{p}<0.05)$. This means that the reconstructed image of prototype DENT-P001 was slightly enlarged, but not distorted as system A.

\subsection{Contrast to noise ratio (CNR)}

CNR presented in prototype system is over three times higher than system A ( $p$ $<0.05$ ) (Table 3).

3.3. Linearity relationship between image intensity and density

The correlation coefficients between image intensity and mineral density in both systems are nearly 1 (Figure 5 ). Here considered the same phantom scanned but the different sizes were obtained by different field of view in two systems. The linear transfer function can help to translate from image intensity to $\mathrm{HU}$, or bone mineral density (BMD) by $\mathrm{mg} / \mathrm{cm}^{2} \mathrm{HA}$. 
A
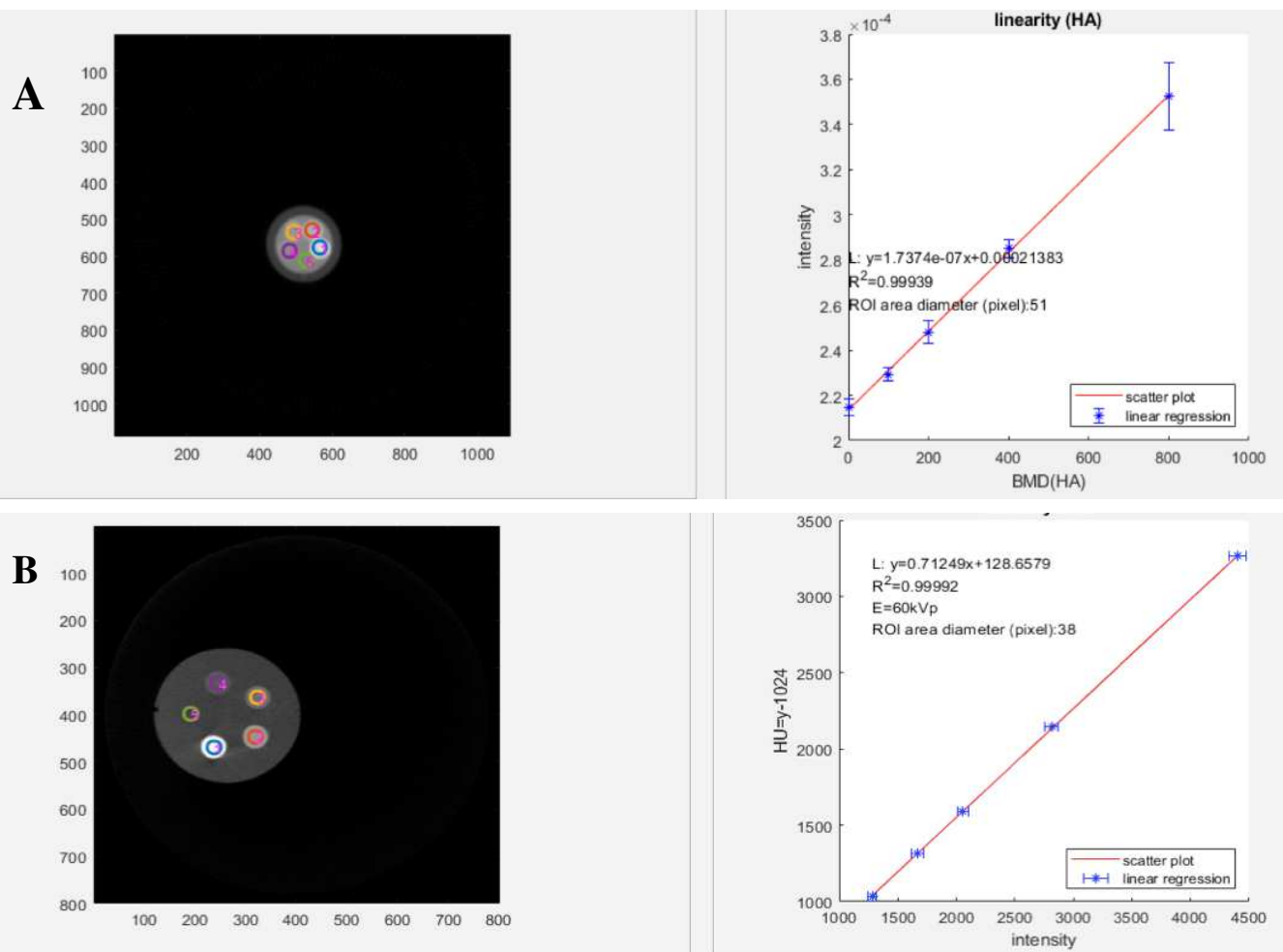

Fig. 5: Central slice of reconstructed HA phantom and analysis's results.

A: Results of prototype DENT-P001 system. B: System A's results.

\subsection{Spatial resolution}

The mean spatial resolutions measuring FWHM of prototype DENT is 0.2446 $\mathrm{mm}$ in and commercial system $\mathrm{A}$ is $0.5179 \mathrm{~mm}$ (Figure 6).

A
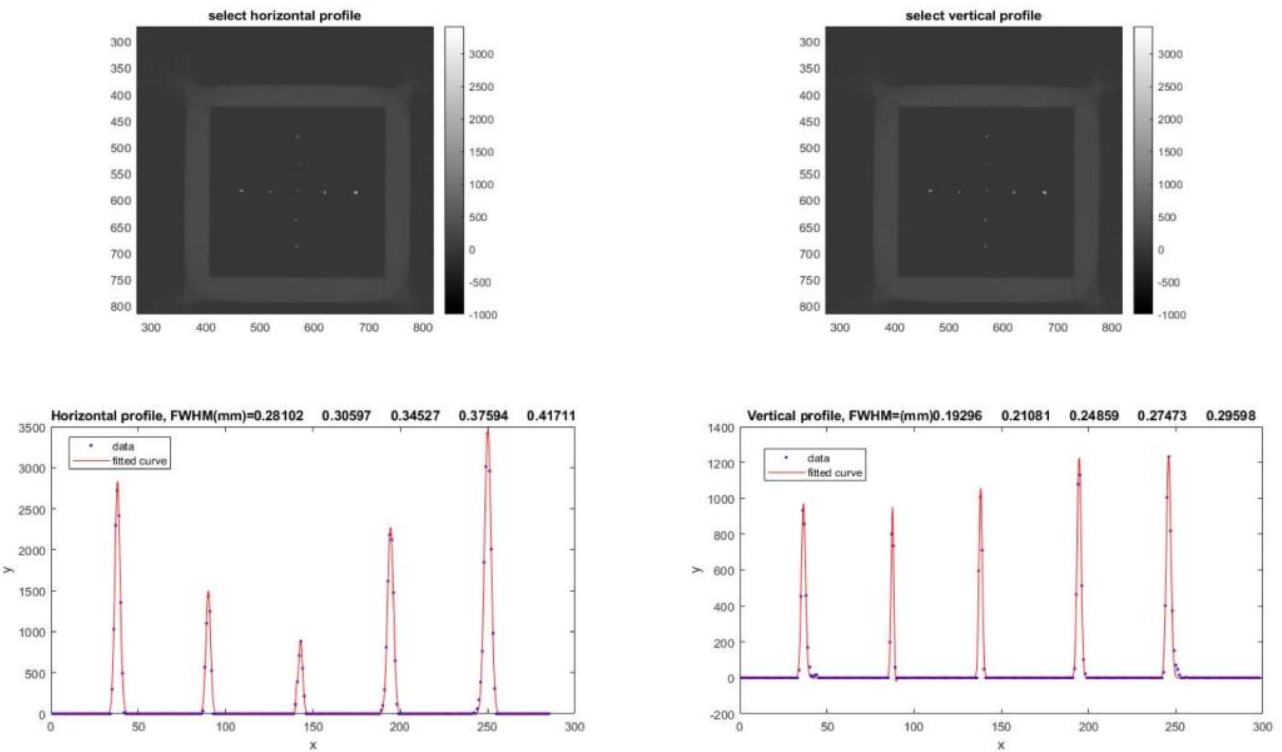

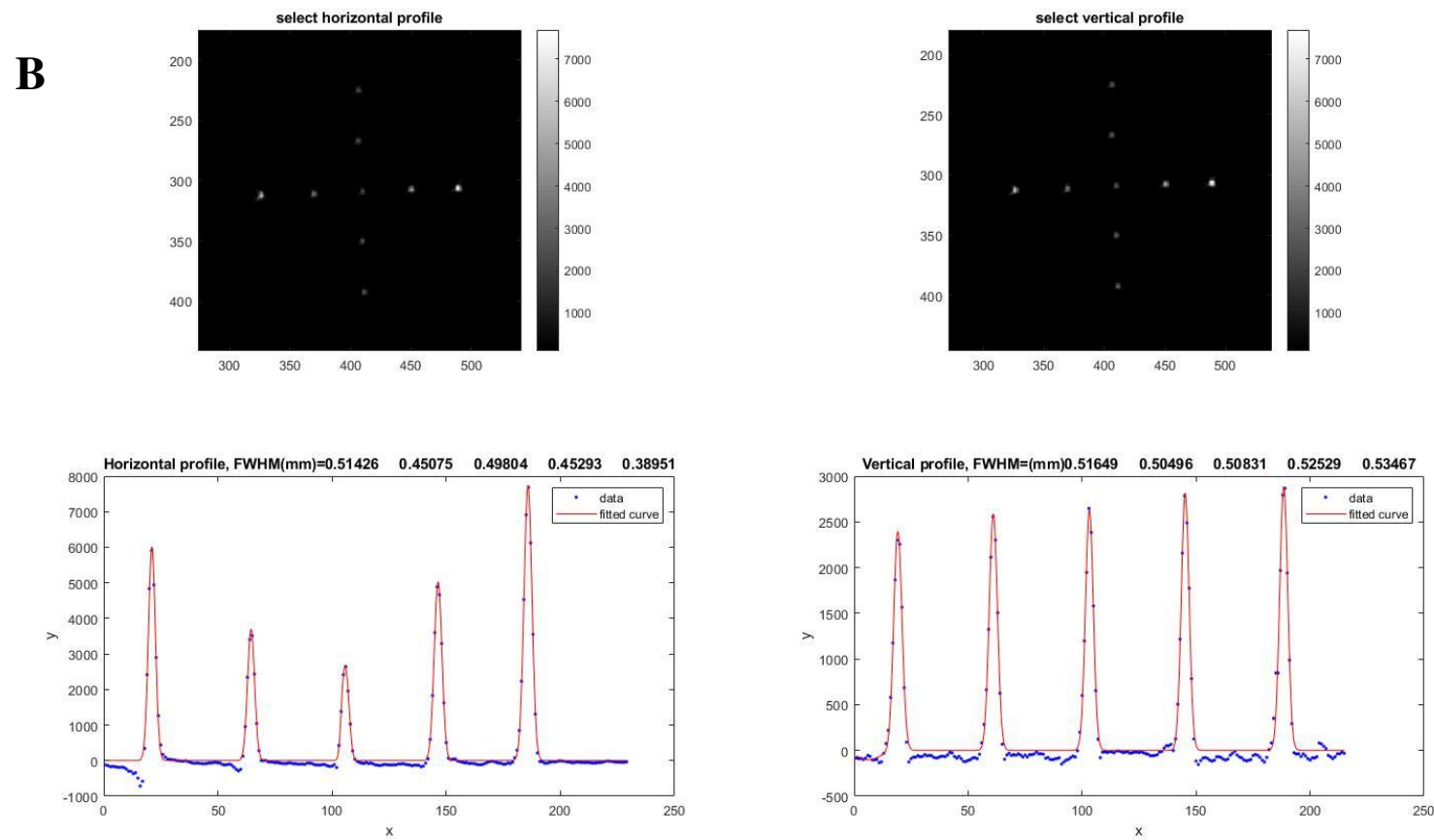

Fig. 6: Central slice of reconstructed wire phantom and analysis's results.

A: Results of prototype DENT-P001 system. B: System A's results.

\section{Discussion}

Table 3: Comparisons of image quality between two systems and factors related $[13,14]$ :

\begin{tabular}{|c|c|c|c|}
\hline & System A & $\begin{array}{l}\text { Prototype } \\
\text { DENT-P001 }\end{array}$ & Can be affected by: \\
\hline CNR* & $100.29 \pm 2.31$ & $329.39 \pm 5.55$ & \multirow{2}{*}{$\begin{array}{l}\text { Dynamic range of the detector, exposure } \\
\text { factors, bit depth, display setting, graphic } \\
\text { card's quality, noise, artifacts. }\end{array}$} \\
\hline Linearity & 0.99992 & $\mathrm{R}=0.99928$ & \\
\hline SNR* & $35.42 \pm 0.61$ & $159.85 \pm 3.88$ & \multirow{2}{*}{$\begin{array}{l}\text { Three sources of noise: } \\
\text { - Quantum noise; } \\
\text { - Electronic noise; } \\
\text { Noise introduced by the } \\
\text { reconstruction process } \\
\text { Be affected by intensity of X-ray } \\
\text { source, circuit, detector design, number of } \\
\text { projections, exposure time, reconstruction } \\
\text { algorithm, etc. }\end{array}$} \\
\hline Uniformity * & $30.59 \pm 2.32$ & $\mathrm{U}=36.34 \pm 0.35$ & \\
\hline Spatial resolution & $0.5179 \mathrm{~mm}$ & $0.2446 \mathrm{~mm}$ & $\begin{array}{l}\text { Focal spot size, detector element size, } \\
\text { number of projections, smoothing filter, }\end{array}$ \\
\hline
\end{tabular}




\begin{tabular}{|c|c|c|l|}
\hline & & $\begin{array}{l}\text { reconstructed voxel size, reconstruction } \\
\text { algorithm. }\end{array}$ \\
\hline $\begin{array}{c}\text { Diameter } \\
\text { difference* }\end{array}$ & $1.59 \pm 0.04 \mathrm{~mm}$ & $1.14 \pm 0.005 \mathrm{~mm}$ & $\begin{array}{l}\text { Distortion correction, center of } \\
\text { rotation correction, flat field correction, } \\
\text { position of the reconstructed slice, } \\
\text { radiation beam geometry, reconstruction } \\
\text { algorithm, the overlap of treatment and } \\
\text { imaging center. }\end{array}$ \\
\hline Distortion* & $0.294 \pm 0.003 \mathrm{~mm}$ & $0.03 \pm 0.002 \mathrm{~mm}$ \\
\hline
\end{tabular}

(*) $p<0.05$

CNR: Contrast to noise ratio; SNR: Signal to noise ratio

In this study, several test phantoms based on tissue or water equivalent materials were chosen with known characteristics and high precision. Thus it is easier to perform constancy and acceptance test of CT imaging systems with these phantoms. QRM Micro-CT Water, QRM Micro - contrast scale and Micro-CT HA are all commercialized and manufactured for standardization among two or more CT imaging systems. Customized wire phantom was designed and fabricated by 3D-printer with small size acupuncture needles. As the limitation of production capacity, the smallest size of needle chosen is $100 \mu \mathrm{m}$, which is less than the reported voxel size of system A $(125 \mu \mathrm{m})$ and as small as prototype's parameter $(100 \mu \mathrm{m})$. The phantoms' sizes were small enough to make sure they are entirely located within the field of view of both systems, so that artifacts related to truncation and error in voxel density value can be eliminated. The objective assessment of image quality using reproducible parameters such as SNR, CNR, uniformity, distortion, spatial resolution, and computer software help simplified the work of technicians in the quality control of CBCT and researchers in comparison among different systems and technologies [14].

Different CT systems, depending on the main application, may be optimized and traded-off between resolution, noise, energy set-up, speed of acquisition stage, and cost. Noise is unavoidable factor impairing radiographic image quality. Noise can be caused from different sources: (1) quantum noise corresponding to the inherent random nature of emission and detection of photons, (2) electronic noise is related to the circuits or the electronics problem in image acquisition stage and (3) noise induced by the reconstruction process [14]. Reducing the noise is obtained by increasing X-ray exposure. Therefore, maximizing SNR is the target when adjusting source energy, while SNR and CNR can be utilized to assess the quality between 
different CT systems [13]. In our study, we eliminated the effect of source energy and window display setting. The detector element sizes of two systems are not far different $(0.15 \mathrm{~mm}$ in system $\mathrm{A}$ and $0.139 \mathrm{~mm}$ in prototype). This difference corresponds to $7,3 \%$ better for prototype. However, result showed that the prototype's image SNR is over five times higher than system A. It means that the number of photons detected by individual detector in prototype system is optimized to reduce the noise, which demonstrates the efficiency of hardware set-up (X-ray tube or detectors) and operation system of prototype DENT-P001. Besides, the effectiveness of software in image processing, denoise, and artifact reduction is also better. In comprison with system A, our prototype applied many correction methods from x-ray spectrum, HU calibration, and artifact reduction base on medical physics (Table 2). Denoise effect has a consequence of blurring and may be presented as the enlarged reconstructed circle (represented as parameter "diameter"). In prototype system, the size and geometry of captured structure is presented more accurately. This may be caused by the smaller voxel size. Moreover, the contrast, which can be compromised by noise reduction or filter algorithm, is still ensured, proving the effective of reconstruction and image processing software of the prototype.

CNR is a quantitative measurement of the low-contrast resolution, which represents for the system's ability to distinguish a signal against certain background. The higher the CNR, the more accurate the ability to differentiate many kinds of tissue. The correlation coefficient between HA concentration and pixel value is presented similarly in both systems $(\mathrm{R} \approx 1)$, but $\mathrm{CNR}$ in prototype is significant better. It might be because prototype system has better noise improvement as mentioned above. When the background and structure have similar attenuation coefficient, the prototype can perform better recognition.

The ROIs chosen for analysis of SNR, uniformity, and CNR were not too small to eliminate the effect of local inhomogeneity and not too big to restrict the impact of artifacts. In the test of attenuation coefficient linearity, the ROIs chosen in each cylindrical is also limited to avoid the blurring edges.

The deformity performed by the prototype is better than system A. The distortion correction or center of rotation correction might be applied differently. However, these are out of the scope of our study. Other factors related to X-ray tube quality, artifacts (X-ray scatter, ring artifact, beam-hardening, truncation, mental artifact) should also be considered and analyzed in future studies.

Spatial resolution is a system-level performance which can be affected by the detector element size, the three-dimensional reconstruction method, the focal spot size, the source-object-detector distances, reconstruction filter, and reconstructed 
voxel size [6]. Two systems used in this study are not much different in terms of detector element size and focal spot size. The better performance in terms of spatial resolution of prototype $(0.2446 \mathrm{~mm})$ compared with system A $(0.5179 \mathrm{~mm}) \mathrm{might}$ be explained by the SOD/ODD ratio of prototype which is higher than system A (2.26 and 2.05, respectively). Besides, the longer source-to-detector distance of prototype (620 mm compared to $520 \mathrm{~mm}$ in system A) allowed higher geometric magnification. Using the modulation transfer function (MTF) approach to quantitatively assess spatial resolution of CBCT systems can verify whether the obtained values are consistent with declared values by manufacturers or not.

\section{Conclusions}

In conclusion, within the limitation of this study, both systems showed acceptable image quality, while prototype DENT-P0001 shows better quality than system A in terms of SNR, CNR, uniformity, spatial resolution, and distortion. The utilization of customized phantom made by technologies and materials in daily dental practice in this study gives suggestion of a simplified phantom design for periodically quality control of CBCT systems.

\section{Acknowledgments}

This research instrument building was supported by funding from the Ministry of Science and Technology, Taiwan, R.O.C grants: MOST 107 - 2328 - 8 - 010 - 003.

\section{REFERENCES}

1. Mozzo P, Procacci C, Tacconi A, Martini PT, Andreis IA. A new volumetric CT machine for dental imaging based on the cone-beam technique: preliminary results. Eur Radiol 1998; 8(9):1558-1564.

2.Popescu D, Laptoiu D. Rapid prototyping for patient-specific surgical orthopaedics guides: A systematic literature review. Proc Inst Mech Eng H. 2016; 230(6): 495-515.

3. Francis Z., Louise R, Mark FM. Image quality assessment tools for optimization of CT images. Radiography 2010; 16(2): 147-153.

4. Liang X, Jacobs R, Hassan B, Li L, Pauwels R, Corpas L et al. A comparative evaluation of Cone Beam Computed Tomography (CBCT) and Multi-Slice CT (MSCT) Part I. On subjective image quality. Eur J Radiol 2010; 75(2): 265-269.

5.Dillenseger JP, Matern JF, Gros CI, Bornert F, Goetz C, Le Minor JM et al., MSCT versus CBCT: evaluation of high-resolution acquisition modes for dento-maxillary and skull-base imaging. Eur. Radiol 2015; 25(2): 505-515.

6.Pauwels R, Araki K, Siewerdsen JH, Thongvigitmanee SS. Technical aspects of dental CBCT: state of the art. Dentomazillofac Radiol 2015; 44, 20140224. 
7. Goldman LW. Principles of CT: radiation dose and image quality. J Nucl Med Technol 2007; 35(4): 213-225.

8.Pauwels R, Beinsberger J, Stamatakis H, Tsiklakis K, Walker A, Bosmans H, et al. Comparison of spatial and contrast resolution for cone-beam computed tomography scanners. Oral Surg Oral Med Oral Pathol Oral Radiol 2012; 114: 127-135.

9. Kim DG. Can dental cone beam computed tomography assess bone mineral density? J Bone Metab 2014; 21(2):117-126.

10. Magill D, Beckmann N, Felice MA, Yoo T, Luo M, Mupparapu M. Investigation of dental cone-beam CT pixel data and a modified method for conversion to Hounsfield unit (HU). Dentomaxillofac Radiol 2018; 47(2): 20170321.

11. Kalender WA, Deak P, Kellermeier M, van Straten M, Vollmar SV. Applicationand patient size-dependent optimization of x-ray spectra for CT. Med Phys 2009; 36, 9931007.

12. QRM, micro-CT \& PET-/SPECT phantoms. Available from https://www.qrm.de/en/overview-pages/micro-ct-pet-spect-phantoms/ [Accessed 22 December 2020].

13. International Organization for Standardization (2017). Non destructive testing Radiation methods for computued tomography - ISO/DIS standard No.15708, part 1-2-3. Retrived from https://www.iso.org/standard/72254.html

14. Las Heras Gala H, Torresin A, Dasu A, Rampado O, Delis H, Hernández Girón I et al. Quality control in cone-beam computed tomography (CBCT) EFOMP-ESTROIAEA protocol (summary report). Phys Med 2017; 39: 67-72. 
Figures

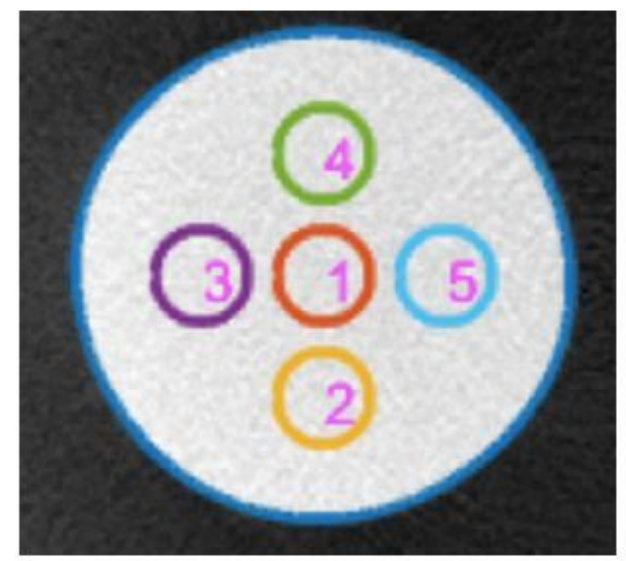

$$
\begin{gathered}
\mathrm{SNR}_{R O I(i)}=\frac{\mu_{R O I(i)}}{\sigma_{R O I(i)}}, \quad i \text { from } 1 \text { to } 5 \\
\mathrm{SNR}=\frac{\sum_{1}^{5} \operatorname{SNR}_{R O I(i)}}{5}
\end{gathered}
$$

\section{Figure 1}

Regions of interest for signal to noise ratio (SNR) \& uniformity's anaslysis: from 1 to 5 ; for distortion determination: outline blue circle

$$
\text { Roundness }=\left|\mathrm{d}_{\max }-\mathrm{d}_{\min }\right|
$$
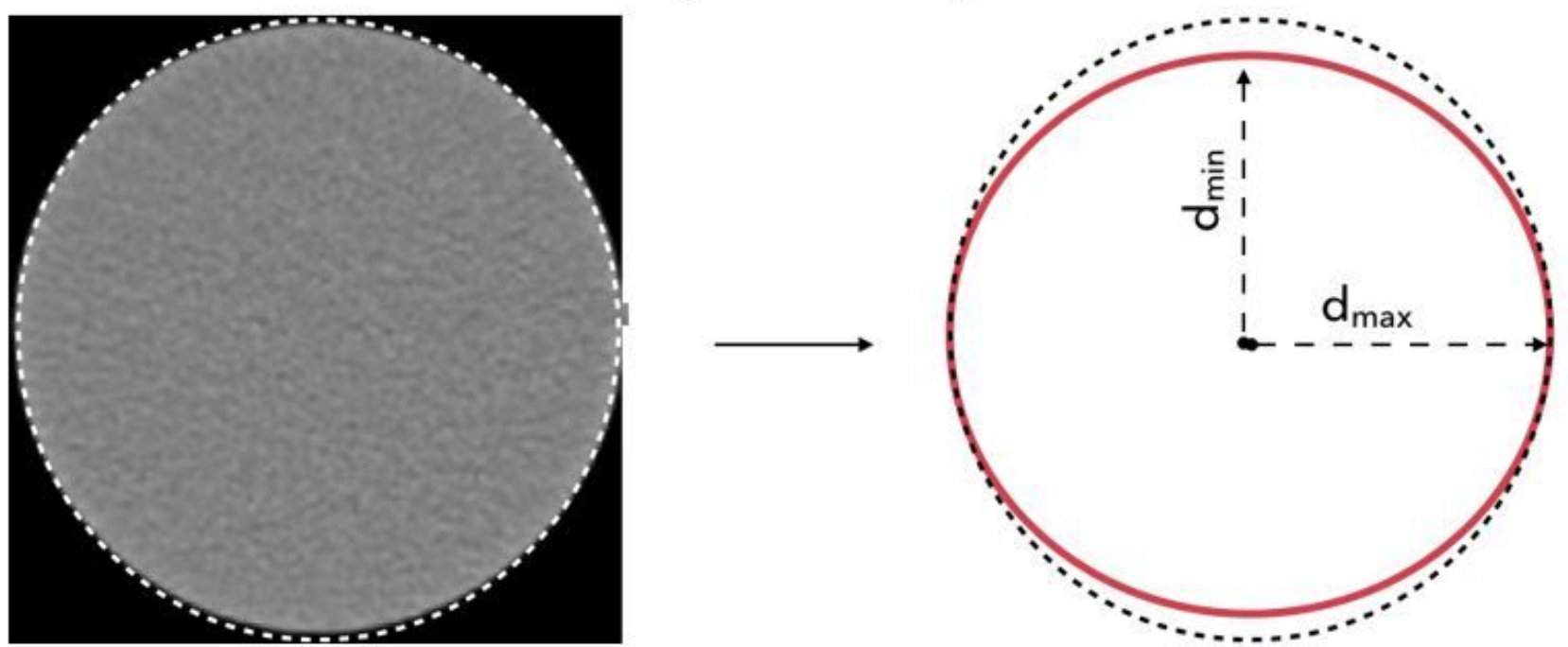

Figure 2

Roundness calculation 


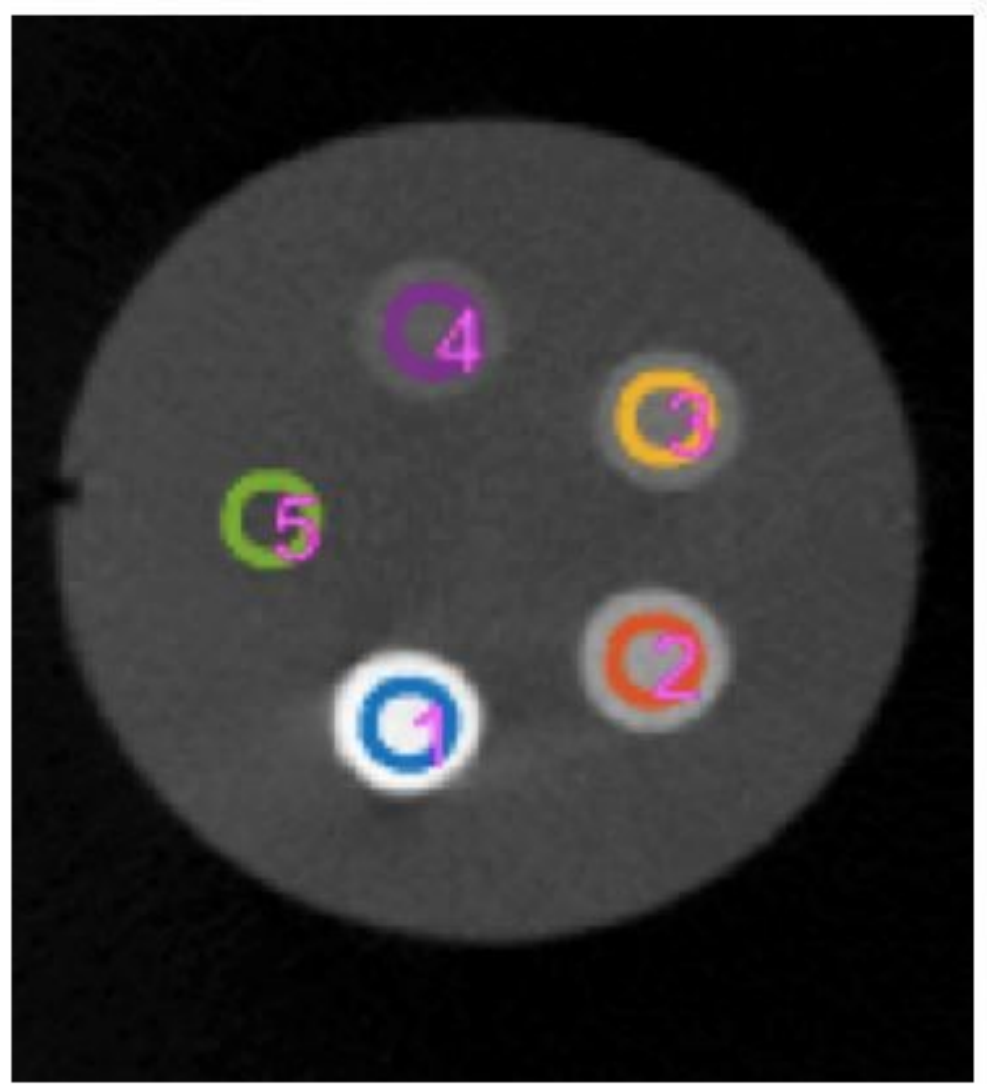

Figure 3

Regions of interest of each hydroxyapatite concentration inserts

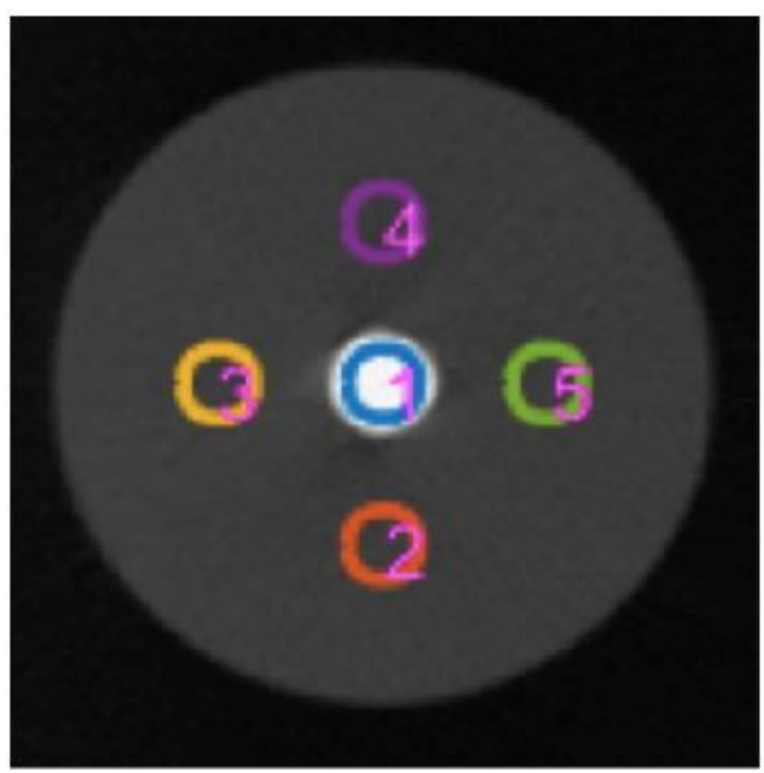

$$
C N R=\frac{\mu_{R O I 1}-\operatorname{mean}\left(\mu_{R O I(i)}\right)}{\sqrt{\sigma_{R O I 1}^{2}+\operatorname{mean}\left(\sigma_{R O I(i)}^{2}\right)}}, i \text { from } 2 \text { to } 5
$$


Figure 4

Contrast to noise determination

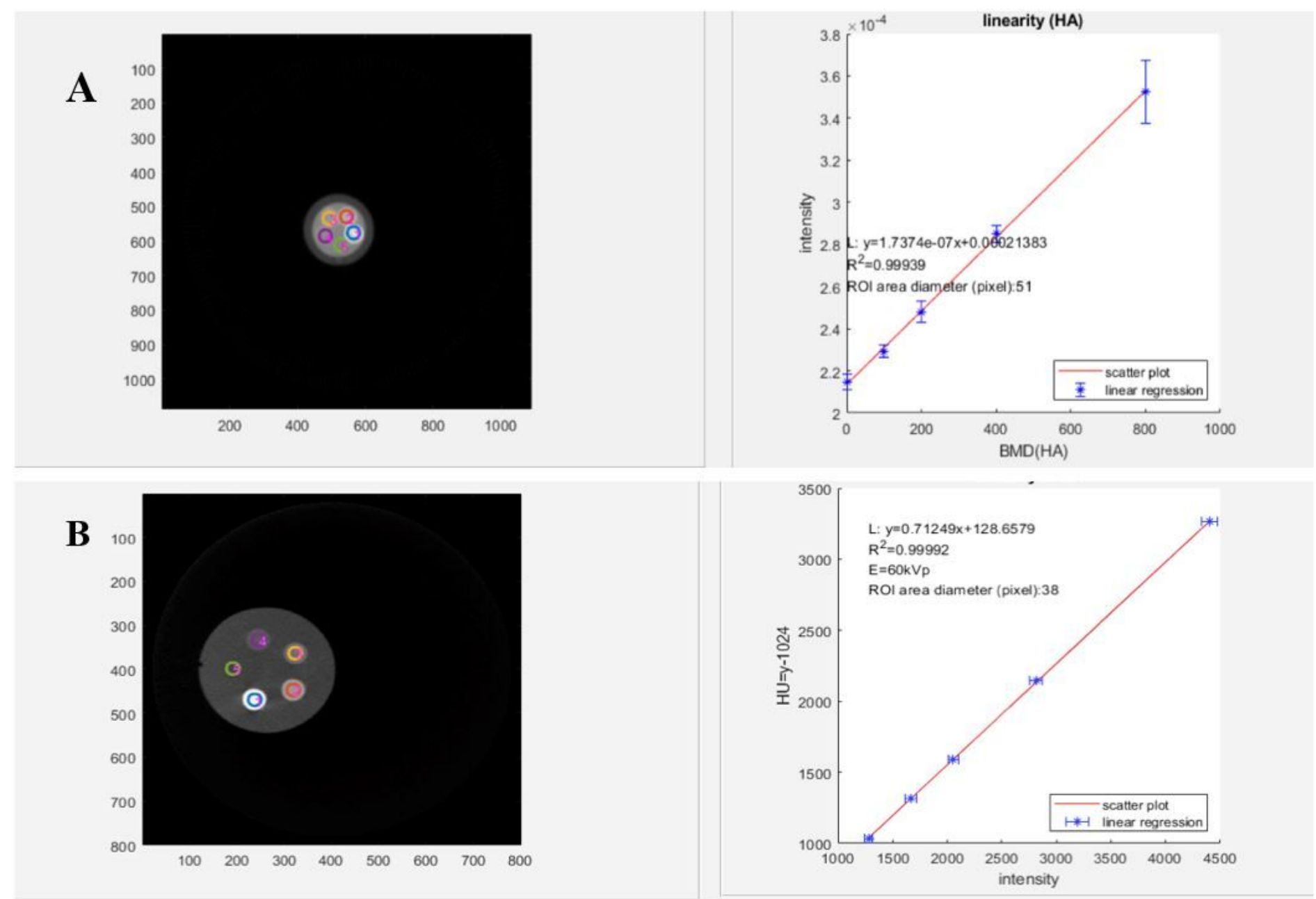

Figure 5

Central slice of reconstructed HA phantom and analysis's results. A: Results of prototype DENT-P001 system. B: System A's results. 
A
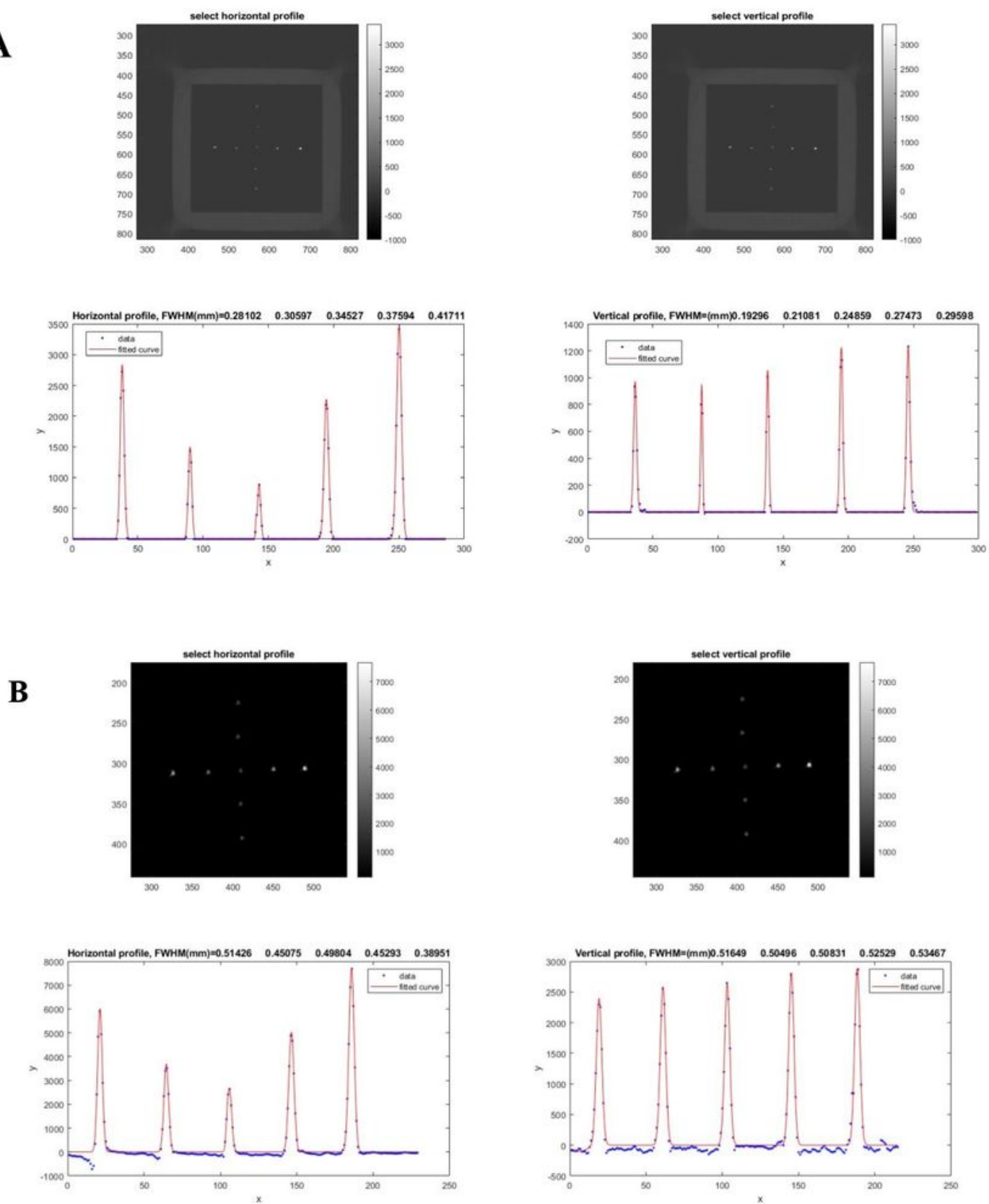

Figure 6

Central slice of reconstructed wire phantom and analysis's results. A: Results of prototype DENT-P001 system. B: System A's results. 